\title{
Semianalytical solutions by homotopy analysis method for EIAV infection with stability analysis
}

\section{S. Geethamalini ${ }^{1}$ and S. Balamuralitharan ${ }^{1 *}$}

\author{
"Correspondence: \\ balamuralitharan.s@ktr.srmuniv.ac.in \\ 'Department of Mathematics, \\ Faculty of Engineering and \\ Technology, SRM Institute of \\ Science and Technology, Tamil \\ Nadu, India
}

\begin{abstract}
Equine Infectious Anemia Virus (EIAV) is a bovine lentivirus group that creates equine infectious anemia in horses around the globe. In this paper, we propose a basic nonlinear differential equation for EIAV infection. Moreover, we obtain a semianalytic approximate solution by the homotopy analysis method (HAM). The gain of this method allows providing a direct scheme for solving the problem. Furthermore, we find the local and global stability of disease-free and endemic equilibrium using a Lyapunov functional. We use Mathematica to carry out the calculations. Graphical outcomes are carried out, and six terms are sufficient to show the effectiveness and power of the method.
\end{abstract}

MSC: $34 \mathrm{G} 20 ; 34 \mathrm{~A} 34$

Keywords: EIAV; HAM; Stability

\section{Introduction}

Equine Infectious Anemia (EIA) is a worldwide infection of lentivirus subfamily of retroviruses, producing fever and anemia. Human immunodeficiency virus (HIV) is also a similar lentivirus subgroup. The resemblances of these infections inspire the investigation of the immune response to EIAV appropriate to study going on HIV. In 1904, the infectious creature that produced EIA was recognized as a filterable agent, assuming that EIA is the animal disease that is allotted a viral etiology [1]. In the 1970s the virus continues lifelong of infected horses in the white blood cells. This lentivirus can be spread by blood sucking biting flies and by the transmission from contaminated needles and improper sterilization of instruments between horses. EIAV is found all through the world with the maximum occurrence in environmental areas with warm atmospheres; henceforth the disease is called a swamp fever [2].

EIAV is the simplest genome of characterized lentiviruses. To control the infection and disease, exposing the working rule of these lentivirus systems has a significant part of persistence and pathogenesis. For the past 30 years, vaccine research for AIDS enduring virus-specific immune responses occuring in inapparent carriers looks for an immunologic regulator of disease by the basic model of EIAV [3].

At first, Nowak and Bangham [4] proposed a mathematical model that includes only immune response CTL. Wodarz [5] suggested a model for cell-mediated and humoral

(c) The Author(s) 2018. This article is distributed under the terms of the Creative Commons Attribution 4.0 International License (http://creativecommons.org/licenses/by/4.0/), which permits unrestricted use, distribution, and reproduction in any medium, provided you give appropriate credit to the original author(s) and the source, provide a link to the Creative Commons license, and indicate if changes were made. 
responses in Hepatitis C virus. Schwartz et al. [6] created a model in which antibody creation is subsidiary amount to virus to regulate the EIAV infection. Ciupe et al. [7] studied a model for EIAV strains and neutralizing antibodies. Schwartz et al. [8] derived a model for EIAV to predict the condition to eradicate the wild-type infection with antibody infusions.

Mathematical model is an essential tool for improvement of our understanding of virus dynamics [9]. Based on review of the literature, till now, there is dearth of knowledge on stability analysis and approximate analytic solutions of EIAV infection. Li and his group analyzed the oscillation criteria for various differential equations [10-13]. Moreover, some approximate solutions are also searched by HAM, which is a powerful method based on the concept of basic topology and shows how to regulate the rate of convergence by allowing an auxiliary parameter $h$ to vary. A suitable choice of the auxiliary linear operator, initial condition, and $h$ ensures the convergence of the HAM solution series. This method was devised by Shijun Liao to solve nonlinear problems. Recently, HAM was used to get the solutions of nonlinear problems of HIV [14-17]. Also, this method gives the solutions of the Klein-Gordon and modified Kawahara equations [18-23]. In the present study, we examine the transmission dynamics of EIAV infection using a mathematical model. We examine the stability of a nonlinear model; also, we find the basic reproduction number and an approximate analytic solution by using HAM.

\section{Basic knowledge of HAM}

Consider the equation

$$
M[v(t)]=0 .
$$

According to the definitions by Liao [14, 15], we give the following zero-order deformation equation:

$$
(1-s) L\left[\phi(t ; s)-v_{0}(t)\right]=\operatorname{sh} H(t) M[\phi(t ; s)], \quad s \in[0,1], h \neq 0,
$$

where $L$ is an auxiliary linear operator such that $L\left[c_{i}\right]=0$ for integral constants $c_{i}(i=$ $1,2,3)$. When $s=0$ and $s=1$, the zero-order deformation equation becomes

$$
\phi(t ; 0)=v_{0}(t), \quad \phi(t ; 1)=v(t) .
$$

By Taylor series expansion of $\phi(t ; s)$ with respect to $s$ we have

$$
\phi(t, s)=v_{0}(t)+\sum_{i=1}^{\infty} v_{i}(t) s^{i},
$$

where

$$
v_{i}=\left.\frac{1}{i !} \frac{\partial^{i} \phi(t ; s)}{\partial s^{i}}\right|_{s=0} .
$$

Differentiating the equation $i$ times with respect to $s$, then fixing $s=0$, and finally dividing them by $i$ !, we get the $i$ th-order deformation equations

$$
L\left[v_{i}(t)-\chi_{i} v_{i-1}(t)\right]=h H(t) R_{i}\left[\vec{v}_{i-1}(t)\right]
$$


where

$$
R_{i}\left[\vec{v}_{i-1}(t)\right]=\left.\frac{1}{(i-1) !} \frac{\partial^{i-1} N[\phi(t ; s)]}{\partial s^{m-1}}\right|_{s=0}
$$

and

$$
\chi_{i}=\left\{\begin{array}{ll}
0 & i \leq 1 \\
1 & i>1
\end{array}\right\} .
$$

These equations can be easily solved using software such as Maple, Matlab, and so on.

\section{Mathematical formulation}

In this paper, we consider the following viral infection models proposed in [4, 24-26]:

$$
\begin{aligned}
& \frac{d E}{d t}=\Lambda-\beta E G-\mu E, \\
& \frac{d F}{d t}=\beta E G-(\omega+\rho) F, \\
& \frac{d G}{d t}=a F-(\psi+\alpha) G .
\end{aligned}
$$

The initial conditions associated with system (1) are

$$
E(0)=E_{0}, \quad F(0)=F_{0} \quad \& \quad G(0)=G_{0} .
$$

A schematic figure is presented in Fig. 1. The parameters and variables are presented in Table 1.

Figure 1 Schematic figure of EIAV infection

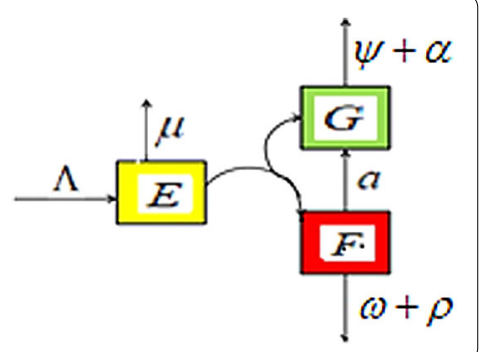

Table 1 Parameter meanings of EIAV infection

\begin{tabular}{ll}
\hline Parameters and variables meaning \\
\hline Dependent variables \\
$E \quad$ susceptible \\
$F \quad$ infected \\
$G \quad$ Virus \\
Parameters and constants \\
$\Lambda \quad$ recruitment rate of susceptible cells \\
$\beta \quad$ rate of infection \\
$\mu \quad$ decay rate of susceptible cells \\
$a \quad$ rate of virus production \\
$\psi \quad$ clearance rate of virus \\
$\alpha \quad$ virus cleared by neutralizing antibodies \\
$\omega$ & death rate of infected cells \\
$\rho$ & effect of infected cell killing by CTLS
\end{tabular}




\section{Mathematical analysis of the EIAV model}

\subsection{Boundedness and positivity}

Theorem 4.1 Let $P:[0,+\infty] \rightarrow R^{3}$. If $P(0) \in R_{+}^{3}$ then $P(t) \in R_{+}^{3}$ for all $t \in[0,+\infty]$, and the solution is $P(t)=(E, F, G)$.

To prove this, note that $\left.\dot{E}\right|_{E=0},\left.\dot{F}\right|_{F=0}$ and $\left.\dot{G}\right|_{G=0}$, are positive for $t>0$. Therefore solutions of model (1) are positive.

Theorem 4.2 Let $P=\left\{(E, F, G) \in R_{+}^{3} \mid 0 \leq E+F+G \leq \bar{E}_{0}, E, F, G \geq 0\right\}$, where $\bar{E}_{0}=\frac{\Lambda}{\mu}$.

Solutions of system (1) are bounded on compact sets. Obviously, solutions of (1) are bounded in the region $P$.

\subsection{Equilibrium analysis}

Disease-free equilibrium and endemic equilibrium:

$$
\begin{aligned}
& D^{0}=\left(E_{0}, F_{0}, G_{0}\right)=\left(\bar{E}_{0}, 0,0\right), \\
& D^{*}=\left(E^{*}, F^{*}, G^{*}\right), \\
& E^{*}=\frac{1}{\beta a}(\omega+\rho)(\psi+\alpha), \\
& F^{*}=\frac{\lambda}{\omega+\rho}-\frac{\mu(\psi+\alpha)}{\beta a}, \\
& G^{*}=\frac{1}{\beta(\omega+\rho)(\psi+\alpha)}[\beta a \Lambda-\mu(\omega+\rho)(\psi+\alpha)] .
\end{aligned}
$$

\subsection{Basic reproduction number}

We find $R_{0}$ using the next generation matrix [27]. Let us take matrices $A$ and $B$ that represent new infection and transfer at disease-free equilibrium,

$$
\begin{aligned}
& A\left(D^{0}\right)=\left[\begin{array}{ll}
0 & \frac{\beta \Lambda}{\mu} \\
0 & 0
\end{array}\right] \text { and } \\
& B\left(D^{0}\right)=\left[\begin{array}{cc}
\omega+\rho & 0 \\
-a & \psi+\alpha
\end{array}\right] . \\
& A\left(D^{0}\right) B\left(D^{0}\right)^{-1}=\left[\begin{array}{cc}
\frac{\beta \Lambda a}{\mu(\omega+\rho)(\psi+\alpha)} & \frac{\beta \Lambda}{\psi+\alpha} \\
0 & 0
\end{array}\right] .
\end{aligned}
$$

Hence

$$
R_{0}=\frac{\beta \Lambda a}{\mu(\omega+\rho)(\psi+\alpha)}
$$

\subsection{Stability analysis}

Proposition 4.3 $D^{0}$ is locally asymptotically stable for $R_{0}<1$ and unstable otherwise. 
Proof

$$
\begin{aligned}
& J=\left[\begin{array}{ccc}
-\mu-\beta G & 0 & -\beta E \\
\beta G & -(\omega+\rho) & \beta E \\
0 & a & -(\psi+\alpha)
\end{array}\right], \\
& J\left(D^{0}\right)=\left[\begin{array}{ccc}
-\mu & 0 & -\frac{\beta \Lambda}{\mu} \\
0 & -(\omega+\rho) & \frac{\beta \Lambda}{\mu} \\
0 & a & -(\psi+\alpha)
\end{array}\right] .
\end{aligned}
$$

Therefore the eigenvalues of the disease-free equilibrium are $-\mu,-(\omega+\rho)$, and $-(\psi+\alpha)$. The eigenvalues at disease-free equilibrium are all negative, and hence it is stable. Therefore if $R_{0}<1$, then $D^{0}$ is locally asymptotically stable.

Proposition 4.4 $D^{0}$ is globally asymptotically stable for $R_{0} \leq 1$ and unstable otherwise.

Proof Let the Lyapunov function $L(E, F, G): R_{+}^{3} \rightarrow R_{+}^{3}$ be defined as

$$
L(E, F, G)=\frac{a F}{\omega+\rho}+G
$$

Differentiating (2) with respect to $t$, we have

$$
\begin{aligned}
\dot{L} & =\frac{a}{\omega+\rho}[\beta E G-(\omega+\rho) F]+a F-(\psi+\alpha) G \\
& \leq \frac{\beta \Lambda a G}{\mu(\omega+\rho)}-(\psi+\alpha) G, \quad E \leq \bar{E}_{0}=\frac{\Lambda}{\mu}, \\
& \leq(\psi+\alpha) G\left(R_{0}-1\right) .
\end{aligned}
$$

This implies $\dot{L} \leq\left(R_{0}-1\right) G \leq 0 \because \dot{L}=0$ only when $G=0$, using $G=0$ in (1) such that $E \rightarrow \frac{\Lambda}{\mu}$ and $F \rightarrow 0$ as $t \rightarrow \infty$. Hence by $[28,29] D^{0}$ is globally asymptotically stable when $R_{0}>1$.

Proposition 4.5 $D^{*}=\left(E^{*}, F^{*}, G^{*}\right)$ is locally asymptotically stable for $R_{0}>1$ and unstable otherwise.

Proof We have

$$
\begin{aligned}
J\left(D^{*}\right) & =\left[\begin{array}{ccc}
\frac{\beta a \Lambda}{(\omega+\rho)(\psi+\alpha)} & 0 & -\frac{(\omega+\rho)(\psi+\alpha)}{(\psi} \\
\frac{\beta a \Lambda}{(\omega+\rho)(\psi+\alpha)}-\mu & -(\omega+\rho) & \frac{(\omega+\rho)(\psi+\alpha)}{a} \\
0 & a & -(\psi+\alpha)
\end{array}\right], \\
J\left(D^{*}\right) & =\left[\begin{array}{ccc}
-\mu R_{0} & 0 & -\frac{(\omega+\rho)(\psi+\alpha)}{a} \\
\mu\left(R_{0}-1\right) & -(\omega+\rho) & \frac{(\omega+\rho)(\psi+\alpha)}{a} \\
0 & a & -(\psi+\alpha)
\end{array}\right] .
\end{aligned}
$$

The characteristic equation of equilibrium $D^{*}$ is

$$
\lambda^{3}+\lambda^{2} M_{1}+\lambda M_{2}+M_{3}=0
$$


where

$$
\begin{aligned}
& M_{1}=\omega+\rho+\alpha+\psi+\frac{(\omega+\rho)(\psi+\alpha)}{a}-\mu R_{0}>0, \\
& M_{2}=\frac{(\omega+\rho)(\psi+\alpha)}{a}\left[\mu R_{0}+\omega+\rho\right]-\mu R_{0}>0, \\
& M_{3}=\frac{(\omega+\rho)^{2}(\psi+\alpha)}{a} \mu R_{0} .
\end{aligned}
$$

By the Routh-Hurwitz criterion

$$
M_{1}>0, M_{3}>0, \quad \text { and } \quad M_{1} M_{2}-M_{3}>0 .
$$

Finally, $D^{*}$ is locally asymptotically stable.

Proposition 4.6 If $R_{0}>1$, then $D^{*}$ is globally asymptotically stable and unstable otherwise.

Proof Consider the Lyapunov function

$$
\begin{aligned}
M\left(E^{*}, F^{*}, G^{*}\right)= & -\left(E^{*} \log \frac{E}{E^{*}}+E^{*}-E\right)-\left(F^{*} \log \frac{F}{F^{*}}+F^{*}-F\right) \\
& -\left(G^{*} \log \frac{G}{G^{*}}+G^{*}-G\right) .
\end{aligned}
$$

Differentiating (3) with respect to $t$, we have

$$
\dot{M}=A \dot{E}+B \dot{F}+C \dot{G}
$$

where

$$
\begin{aligned}
A E= & E^{*}-E, \quad B F=F^{*}-F, \quad C G=G^{*}-G, \quad Q E=E^{*}, \\
\dot{M}= & \Lambda+A^{2} E \beta G^{*}+B \beta G E+B \beta G^{*} E^{*} \\
& +C\left[a F+\alpha G^{*}+\psi G^{*}\right]-\Lambda Q-A^{2} E \beta G \\
& -A^{2} E \mu-B \beta G^{*} G-B \beta G E^{*}-B^{2} F(\omega+\rho)-C a F^{*}-(\alpha+\psi) C G, \\
\dot{M}= & U-V,
\end{aligned}
$$

where

$$
\begin{aligned}
U= & \Lambda+A^{2} E \beta G^{*}+B \beta G E+B \beta G^{*} E^{*} \\
& +C\left[a F+\alpha G^{*}+\psi G^{*}\right], \\
V= & \Lambda Q+A^{2} E \beta G+A^{2} E \mu+B \beta G^{*} G+B \beta G E^{*}+B^{2} F(\omega+\rho)+C a F^{*}+(\alpha+\psi) C G .
\end{aligned}
$$

From (4) we have that if $U<V$, then $\dot{M} \leq 0$. Also, $\dot{M}=0$ iff $E^{*}=E, F^{*}=F, G^{*}=G$. Hence by $[28,29] D^{*}$ is globally asymptotically stable when $R_{0}>1$. 


\section{Application}

To construct the solutions of (1) by HAM, we first choose

$$
E(0)=E_{0}, \quad F(0)=F_{0}, \quad G(0)=G_{0} .
$$

We choose the auxiliary linear operators $L_{1}, L_{2}$, and $L_{3}$ as

$$
\begin{aligned}
& L_{1}[E(t, s)]=\frac{d E(t, s)}{d t}, \\
& L_{2}[F(t, s)]=\frac{d F(t, s)}{d t}, \\
& L_{3}[G(t, s)]=\frac{d G(t, s)}{d t} .
\end{aligned}
$$

They satisfy the following properties:

$L_{i}\left(C_{i}\right)=0$, where $C_{i}(i=1,2,3)$ are integral constants. Define the nonlinear operators

$$
\begin{aligned}
& N_{1}[E, F, G]=\dot{E}-\Lambda+\beta E G+\mu E, \\
& N_{2}[E, F, G]=\dot{F}-\beta E G+(\omega+\rho) F, \\
& N_{3}[E, F, G]=\dot{G}-a F+(\psi+\alpha) G .
\end{aligned}
$$

By the definitions of Liao $[14,15]$ introduce the nonzero auxiliary parameter $h$, nonzero auxiliary function $\mathrm{H}(\mathrm{t})$, and the embedding parameter $s$ in $[0,1]$. Then using this, we form the zero-order deformation equations

$$
\begin{aligned}
& (1-s) L_{1}\left[E(t ; s)-E_{0}(t)\right]=s h_{1} H_{1}(t) N_{1}[E, F, G], \\
& (1-s) L_{2}\left[F(t ; s)-F_{0}(t)\right]=s h_{2} H_{2}(t) N_{2}[E, F, G], \\
& (1-s) L_{3}\left[G(t ; s)-G_{0}(t)\right]=s h_{3} H_{3}(t) N_{3}[E, F, G],
\end{aligned}
$$

clearly, when $s=0$ and $s=1$, we have

$$
\begin{array}{ll}
E(t ; 0)=E_{0}(t), & E(t ; 1)=E(t), \\
F(t ; 0)=F_{0}(t), & F(t ; 1)=F(t), \\
G(t ; 0)=G_{0}(t), & G(t ; 1)=G(t) .
\end{array}
$$

Thus the embedding parameter s rises from zero to one, the solutions $E(t ; s), F(t ; s)$, and $G(t ; s)$ vary continuously from $E_{0}(t), F_{0}(t)$, and $G_{0}(t)$ to the exact solution $E(t), F(t)$, and $G(t)$. Expanding $E(t ; s)$ by Taylor's series, we have

$$
\begin{aligned}
& E(t ; s)=E_{0}(t)+\sum_{i=1}^{\infty} E_{i}(t) s^{i}, \\
& F(t ; s)=F_{0}(t)+\sum_{i=1}^{\infty} F_{i}(t) s^{i}, \\
& G(t ; s)=G_{0}(t)+\sum_{i=1}^{\infty} G_{i}(t) s^{i},
\end{aligned}
$$


where

$$
\begin{aligned}
& X_{i}=\left.\frac{1}{i !} \frac{\partial^{i} E(t ; s)}{\partial s^{i}}\right|_{s=0}, \\
& F_{i}=\left.\frac{1}{i !} \frac{\partial^{i} F(t ; s)}{\partial s^{s}}\right|_{s=0}, \\
& G_{i}=\left.\frac{1}{i !} \frac{\partial^{i} G(t ; s)}{\partial s^{i}}\right|_{s=0} .
\end{aligned}
$$

If $h_{1}, h_{2}, h_{3}, H_{1}(t), H_{2}(t)$, and $H_{3}(t)$ are chosen, then the series is convergent at $p=1$.

$$
\begin{aligned}
& E(t)=E_{0}(t)+\sum_{i=1}^{\infty} E_{i}(t), \\
& F(t)=F_{0}(t)+\sum_{i=1}^{\infty} F_{i}(t), \\
& Z(t)=Z_{0}(t)+\sum_{i=1}^{\infty} Z_{i}(t) .
\end{aligned}
$$

Differentiating (6)-(8) $i$ times with respect to $s$, allocating by $i$ !, and fixing $s=0$, the $i$ th-order deformation equations are

$$
\begin{aligned}
& L_{1}\left[E_{i}(t)-\chi_{i} E_{i-1}(t)\right]=h R_{1, i}\left(E_{i-1}(t)\right), \\
& L_{2}\left[F_{i}(t)-\chi_{i} F_{i-1}(t)\right]=h R_{2, i}\left(F_{i-1}(t)\right), \\
& L_{3}\left[G_{i}(t)-\chi_{i} G_{i-1}(t)\right]=h R_{3, i}\left(G_{i-1}(t)\right),
\end{aligned}
$$

where

$$
\begin{aligned}
& R_{1, i}(t)=\frac{d E_{i-1}(t)}{d t}+\beta \sum_{j=0}^{i-1} E_{j}(t) G_{i-1-j}(t)+\mu E_{i-1}(t)-\left(1-\chi_{i}\right) \Lambda, \\
& R_{2, i}(t)=\frac{d F_{i-1}(t)}{d t}-\beta \sum_{j=0}^{i-1} G_{j}(t) E_{i-1-j}(t)+(\omega+\rho) F_{i-1}(t), \\
& R_{3, i}(t)=\frac{d G_{i-1}(t)}{d t}-a F_{i-1}(t)+(\psi+\alpha) Z_{i-1}(t),
\end{aligned}
$$

and

$$
\chi_{i}=\left\{\begin{array}{ll}
0 & i \leq 1 \\
1 & i>1
\end{array}\right\} .
$$

Then the $i$ th-order deformation (18)-(20) for $i$ greater than or equal to 1 becomes

$$
\begin{aligned}
& E_{i}(t)=\chi_{i} E_{i-1}(t)+h \int_{0}^{t} R_{1, i}(\tau) d \tau, \\
& F_{i}(t)=\chi_{i} F_{i-1}(t)+h \int_{0}^{t} R_{2, i}(\tau) d \tau, \\
& G_{i}(t)=\chi_{i} G_{i-1}(t)+h \int_{0}^{t} R_{3, i}(\tau) d \tau .
\end{aligned}
$$




\section{Numerical results}

Consider the following values for numerical results [30]:

$$
\begin{aligned}
& E_{0}=42,390, \quad F_{0}=0, \quad G_{0}=0.001, \\
& \Lambda=2019, \quad \beta=0.00000094, \quad \mu=0.048, \\
& a=101, \quad \psi=2.33, \quad \alpha=3, \quad \omega=0.057, \quad \rho=0.01 .
\end{aligned}
$$

We use Mathematica software to get the sixth-order expansions for $E(t), F(t)$, and $G(t)$ :

$$
\begin{aligned}
E(t)= & 42,390+94.32 h t+235.8 h^{2} t+314.4 h^{3} t+235.8 h^{4} t \\
& +94.32 h^{5} t+15.72 h^{6} t+5.65936 h^{2} t^{2}+15.0916 h^{3} t^{2}+16.97814 h^{4} t^{2} \\
& +9.05498 h^{5} t^{2}+1.88645 h^{6} t^{2}+0.121116 h^{3} t^{3}+0.27251 h^{4} t^{3}+\cdots \\
F(t)= & -0.000023908 h t-0.0000597699 h^{2} t-0.000079632 h^{3} t \\
& -0.0000597699 h^{4} t-0.000023908 h^{5} t-0.0000098466 h^{6} t \\
& -0.0001613 h^{2} t^{2}-0.000430134 h^{3} t^{2}-0.0004839 h^{4} t^{2} \\
& -0.00025808 h^{5} t^{2}-0.0000537667 h^{6} t^{2}-0.000387534 h^{3} t^{3} \\
& -0.0000871952 h^{4} t^{3}-0.000697561 h^{5} t^{3}-0.000193767 h^{6} t^{3}-\cdots \\
G(t)= & 0.001+0.03198 h t+0.07995 h^{2} t+0.1066 h^{3} t+0.07995 h^{4} t \\
& +0.03198 h^{5} t+0.00533 h^{6} t+0.216085 h^{2} t^{2}+0.576227 h^{3} t^{2} \\
& +0.648255 h^{4} t^{2}+0.345736 h^{5} t^{2}+0.0720284 h^{6} t^{2}+0.519122 h^{3} t^{3} \\
& +1.16803 h^{4} t^{3}+0.93442 h^{5} t^{3}+0.0720284 h^{6} t^{3}+\cdots
\end{aligned}
$$

\section{Discussion}

We have found the solution of (18)-(19) containing $h$ by showing an easy technique for control and adjustment of curves ensuring the convergence of solution series, as recommended by Liao. Figures $2-7$ show the plots of fifth and sixth term approximation of $E^{\prime}(0)$, $F^{\prime}(0)$ and $G^{\prime}(0)$.

These curves show that the valid region of $h$ is parallel to the horizontal axis. The valid region is listed in Table 2.

Figure 2 The $h$-curves of $E^{\prime}(0)$ obtained by 5th-order approximation of the HAM

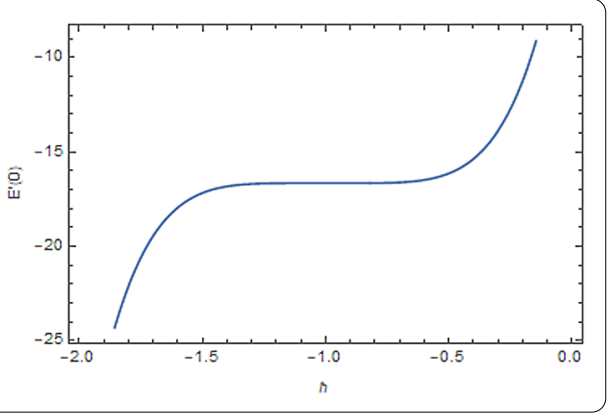


Figure 3 The $h$-curves of $F^{\prime}(0)$ obtained by 5 th-order approximation of the HAM

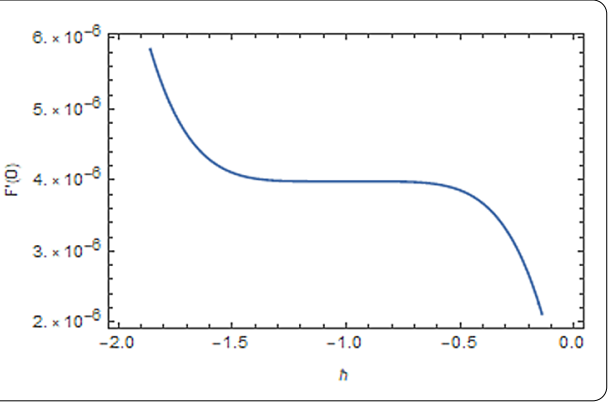

Figure 4 The $h$-curves of $G^{\prime}(0)$ obtained by 5th-order approximation of the HAM

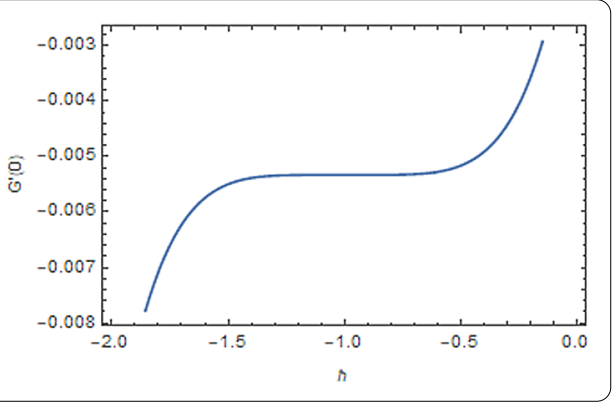

Figure 5 The $h$-curves of $E^{\prime}(0)$ obtained by 6 th-order approximation of the HAM

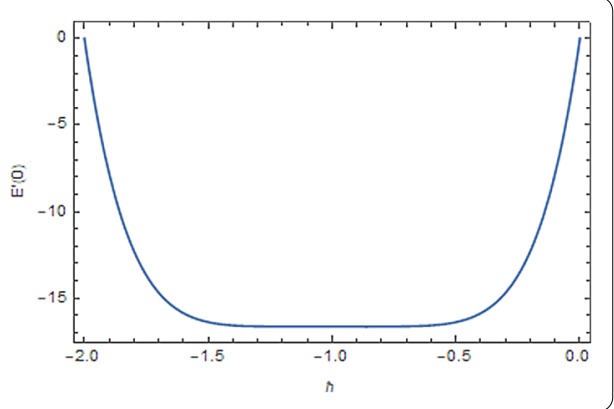

Figure 6 The $h$-curves of $F^{\prime}(0)$ obtained by 6 th-order approximation of the HAM

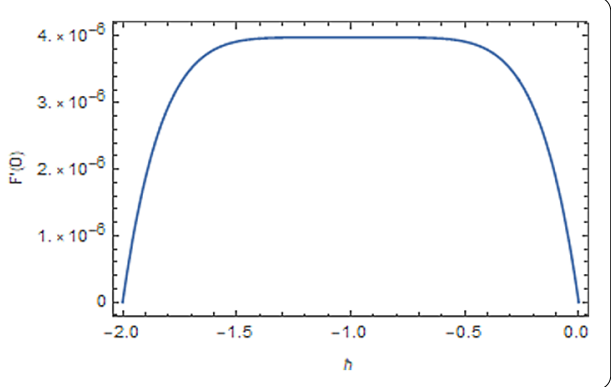

In this context, an error analysis is performed to get the optimal values of $h$. We substitute Eqs. (21)-(23) into (1) and obtain the residual functions as follows:

$$
\begin{aligned}
& E R_{1}\left(E, F, G ; h_{1}\right)=\frac{d \phi_{E}\left(t ; h_{1}\right)}{d t}-\Lambda+\beta \phi_{E}\left(t ; h_{1}\right) \phi_{G}\left(t ; h_{1}\right)+\mu \phi_{E}\left(t ; h_{1}\right), \\
& E R_{2}\left(E, F, G ; h_{2}\right)=\frac{d \phi_{F}\left(t ; h_{2}\right)}{d t}-\beta \phi_{E}\left(t ; h_{2}\right) \phi_{G}\left(t ; h_{2}\right)+(\omega+\rho) \phi_{F}\left(t ; h_{2}\right), \\
& E R_{3}\left(E, F, G ; h_{3}\right)=\frac{d \phi_{G}\left(t ; h_{3}\right)}{d t}-a \phi_{F}\left(t ; h_{3}\right)-(\psi+\alpha) \phi_{F}\left(t ; h_{3}\right) .
\end{aligned}
$$


Figure 7 The $h$-curves of $G^{\prime}(0)$ obtained by

Figure 7 The $h$-curves of $G^{\prime}(0)$ obtain
6th-order approximation of the HAM

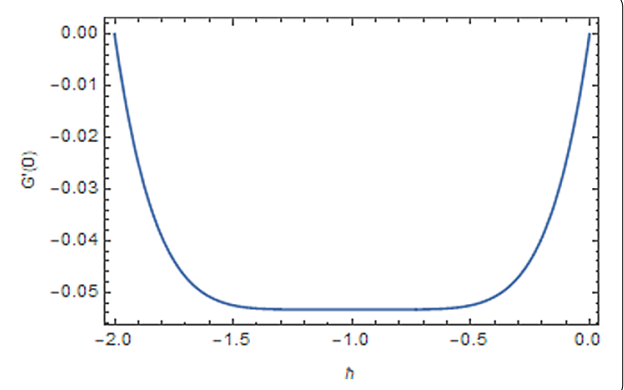

Table 2 Figures 2-7 show the values of $h$

\begin{tabular}{ll}
\hline$E(t)$ & $-1.35 \leq h \leq-0.6$ \\
$F(t)$ & $-1.4 \leq h \leq-0.7$ \\
$G(t)$ & $-1.4 \leq h \leq-0.7$ \\
\hline
\end{tabular}

Table 3 The minimum values of $R E\left(h_{1}^{*}\right), R F\left(h_{2}^{*}\right), R G\left(h_{3}^{*}\right)$ (see Figs. 11-13)

\begin{tabular}{lll}
\hline & $h^{*}$ & Minimum value \\
\hline$R E\left(h_{1}\right)$ & -0.906678 & $2.227296 \times 10^{-4}$ \\
$R F\left(h_{2}\right)$ & -0.660648 & $1.32472 \times 10^{-15}$ \\
$R G\left(h_{3}\right)$ & -0.660667 & $2.37742 \times 10^{-9}$ \\
\hline
\end{tabular}

Following [31, 32], we consider the square residual error for the sixth-order approximation:

$$
\begin{aligned}
& R E\left(h_{1}\right)=\int_{0}^{1}\left(E R_{1}\left(E, F, G ; h_{1}\right)\right)^{2} d t, \\
& R F\left(h_{2}\right)=\int_{0}^{1}\left(E R_{2}\left(E, F, G ; h_{2}\right)\right)^{2} d t, \\
& R G\left(h_{3}\right)=\int_{0}^{1}\left(E R_{3}\left(E, F, G ; h_{3}\right)\right)^{2} d t .
\end{aligned}
$$

The values of $h_{1}, h_{2}$, and $h_{3}$ for which $R E\left(h_{1}\right), R F\left(h_{2}\right), R G\left(h_{3}\right)$ are minimal can be obtained. We have

$$
\frac{d R E\left(h_{1}^{*}\right)}{d h_{1}}=0, \quad \frac{d R F\left(h_{2}^{*}\right)}{d h_{2}}=0, \quad \frac{d R G\left(h_{3}^{*}\right)}{d h_{3}}=0 .
$$

The optimal values of $h_{1}, h_{2}$, and $h_{3}$ for all of the cases considered are

$$
h_{1}^{*}=-0.906678, \quad h_{2}^{*}=-0.660648, \quad h_{3}^{*}=-0.660667 .
$$

In Table 3, the minimum values of $R E\left(h_{1}\right), R F\left(h_{2}\right)$, and $R G\left(h_{3}\right)$ are given for optimal values of $h_{1}, h_{2}$, and $h_{3}$.

In Table 4, we calculated errors $E R_{1}, E R_{2}$, and $E R_{3}$ for various $t$ in $(0,1)$. This shows that the HAM offers us an accurate approximate solution for EIAV model (1).

Figures 8-10 show the residual errors of $E R_{1}, E R_{2}$, and $E R_{3}$ for $t$ in $(0,1)$ and various $h$. By considering the following figures note the solutions obtained by using HAM. 
Table 4 The residual errors $E R_{1}, E R_{2}$, and $E R_{3}$ for various $t \in(0,1)$

\begin{tabular}{llll}
\hline$t$ & $E R_{1}\left(E, F, G ; h_{1}^{*}\right)$ & $E R_{2}\left(E, F, G ; h_{2}^{*}\right)$ & $E R_{3}\left(E, F, G ; h_{3}^{*}\right)$ \\
\hline 0.0 & $1.03838 \times 10^{-5}$ & $6.08546 \times 10^{-9}$ & $8.13736 \times 10^{-6}$ \\
0.1 & $7.64421 \times 10^{-6}$ & $1.13927 \times 10^{-9}$ & $1.53872 \times 10^{-6}$ \\
0.2 & $5.2269 \times 10^{-6}$ & $5.09644 \times 10^{-9}$ & $6.81296 \times 10^{-6}$ \\
0.3 & $3.09924 \times 10^{-6}$ & $1.78769 \times 10^{-9}$ & $2.40852 \times 10^{-6}$ \\
0.4 & $1.27796 \times 10^{-6}$ & $1.28159 \times 10^{-8}$ & $1.71806 \times 10^{-5}$ \\
0.5 & $1.38351 \times 10^{-7}$ & $9.88247 \times 10^{-9}$ & $1.32496 \times 10^{-5}$ \\
0.6 & $8.18731 \times 10^{-7}$ & $1.60802 \times 10^{-8}$ & $2.15323 \times 10^{-5}$ \\
0.7 & $1.95882 \times 10^{-8}$ & $5.51901 \times 10^{-8}$ & $7.39272 \times 10^{-5}$ \\
0.8 & $3.65462 \times 10^{-6}$ & $7.69584 \times 10^{-8}$ & $1.03087 \times 10^{-4}$ \\
0.9 & $1.04486 \times 10^{-3}$ & $3.68833 \times 10^{-8}$ & $4.93837 \times 10^{-5}$ \\
1 & $3.02903 \times 10^{-5}$ & $1.08705 \times 10^{-7}$ & $1.45783 \times 10^{-4}$ \\
\hline
\end{tabular}

Figure 8 The residual errors function Eq. (24) using the 6th-order approximation solution for various $h=-0.9, h=-1, h=$ optimal, $h=-1.1$ and $t \in(0,1)$

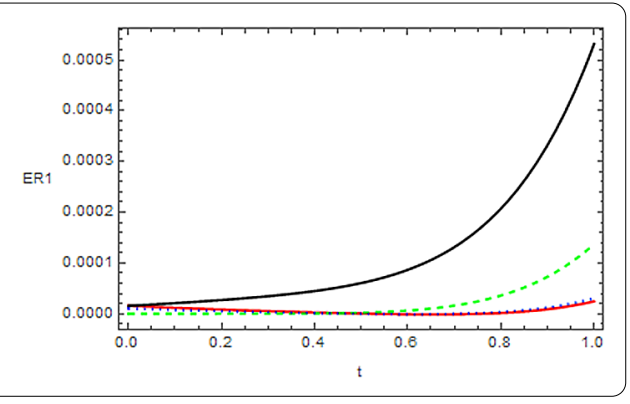

Figure 9 The residual error function Eq. (25) using the 6 th-order approximation solution for various $h=-0.9, h=-1, h=$ optimal, $h=-1.1$ and $t \in(0,1)$

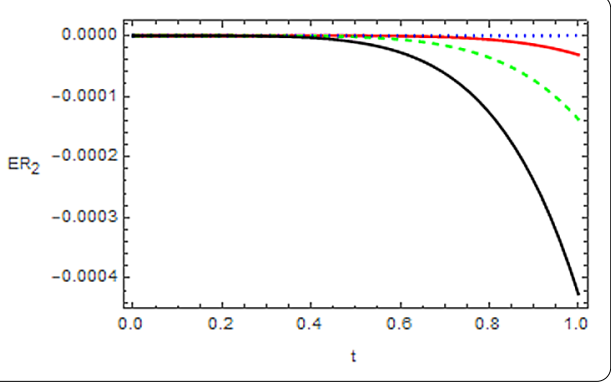

Figure 10 The residual error function Eq. (26) using the 6th-order approximation solution for various $h=-0.9, h=-1, h=$ optimal, $h=-1.1$ and $t \in(0,1)$

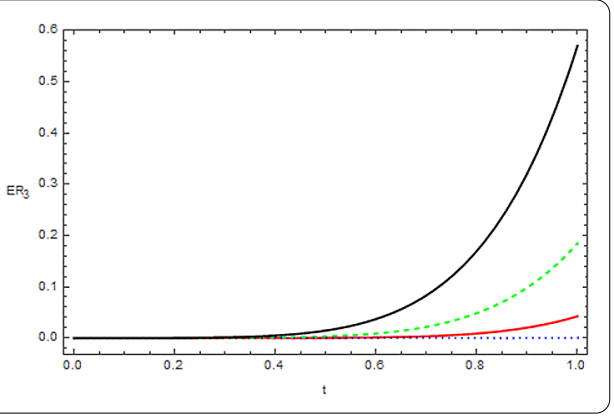

\section{Conclusion}

In this paper, we examined the global dynamics by $R_{0}$. The disease-free equilibrium is globally asymptotically stable if $R_{0} \leq 1$. Moreover, the endemic equilibrium is globally asymptotically stable if $R_{0}>1$. Also, we obtain an approximate analytical solution for this model. Moreover, we illustrate the ability of HAM to confirm the convergence of sequence 
Figure 11 The optimum and minimum values of $E(t)$

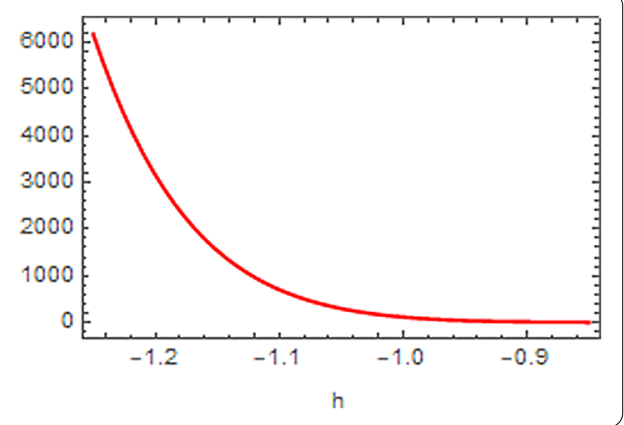

Figure 12 The optimum and minimum values of $F(t)$

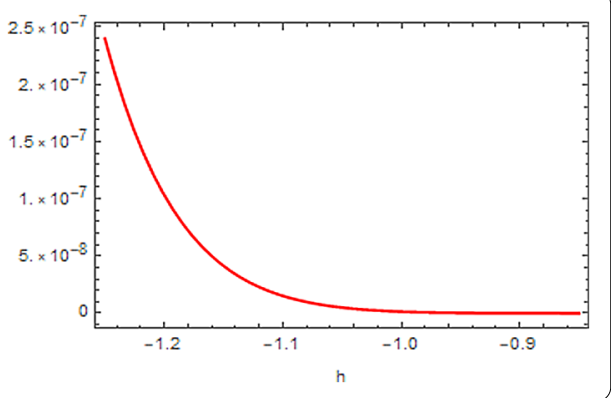

Figure 13 The optimum and minimum values of $G(t)$

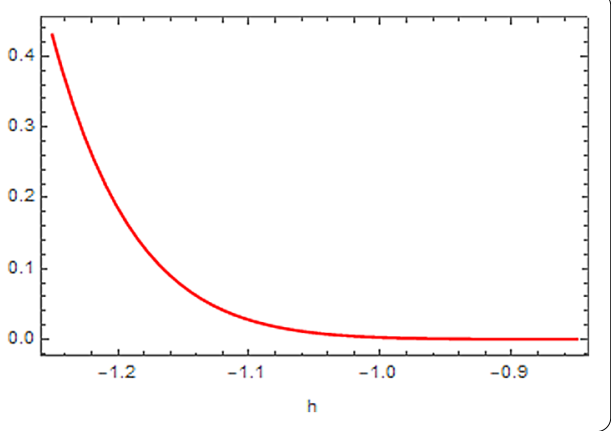

solution for nonlinear differential equations and obtain approximate semianalytic solutions. In this manner, HAM is very effective and powerful technique to find the solutions.

\section{Acknowledgements}

The authors are grateful to anonymous referees for their excellent suggestions, which greatly improved the presentation of the paper.

\section{Funding}

The financial assistance from the SRM Institute of Science and Technology, Chennai, in the form of a University Research fellowship (URF) is gratefully acknowledged.

Availability of data and materials

Data sharing not applicable to this article as no data sets were generated or analyzed during the current study.

\section{Competing interests}

The authors declare that there is no conflict of interests.

Authors' contributions

Both authors contributed equally to the writing of this paper. Both authors read and approved the final manuscript. 


\section{Publisher's Note}

Springer Nature remains neutral with regard to jurisdictional claims in published maps and institutional affiliations.

\section{Received: 2 July 2018 Accepted: 18 September 2018 Published online: 04 October 2018}

\section{References}

1. Leroux, C., Cadore, J.L., Montelaro, R.C.: Equine Infectious Anemia Virus (EIAV) what has HIV's country cousin got to tell us? Vet. Res. 35, 485-512 (2004)

2. Cook, R.F., Leroux, C., Issel, C.J.: Equine infectious anemia and equine infectious anemia virus in a review. Vet. Microbiol. 167, 181-204 (2013)

3. Issel, C.J., Cook, R.F., Mealey, R.H., Horohov, D.W.: Equine infectious anemia in 2014: live with it or eradicate it? Vet. Clin. North Am., Equine Pract. 30(3), 561-577 (2014)

4. Nowak, A., Bangham, C.R.: Population dynamics of immune responses to persistent viruses. Science 272(5258), 74-79 (1996)

5. Wodarz, D.: Hepatitis C virus dynamics and pathology: the role of CTL and antibody responses. J. Gen. Virol. 84, 1743-1750 (2003)

6. Schwartz, E.J., Pawelek, K.A., Harrington, K., Cangelosi, R., Madrid, S.: Immune control of equine infectious anemia virus infection by CTLs and antibodies. Appl. Math. 4, 171-177 (2013)

7. Ciupe, S.M., Schwartz, E.J.: Understanding virus host dynamics following EIAV infection in SCID horses. J. Theor. Biol. $343,1-8(2014)$

8. Schwartz, E.J., Smith, R.J.: Identifying the conditions under which antibodies protect against infection by equine infectious anemia virus. Vaccines 2(2), 397-421 (2014)

9. Anderson, R.M., May, R.M.: Infectious Diseases of Humans. Dynamics and Control. Oxford University Press, Oxford (1991)

10. Bohner, M., Hassan, T.S., Li, T.: Fite-Hille-Wintner-type oscillation criteria for second-order half-linear dynamic equations with deviating arguments. Indag Math. 29, 548-560 (2018)

11. Chatzarakis, G.E., Li, T.: Oscillation criteria for delay and advanced differential equations with nonmonotone arguments. Complexity 2018, Article ID 8237634 (2018)

12. Chatzarakis, G.E., Li, T.: Oscillations of differential equations generated by several deviating arguments. Adv. Differ. Equ. 2017, Article ID 292 (2017)

13. Li, T., Rogovchenko, Yu.V:: Oscillation criteria for second-order superlinear Emden-Fowler neutral differential equations. Monatshefte Math. 184, 489-500 (2017)

14. Liao, S.J.: Notes on the homotopy analysis method: some definitions and theorems. Commun. Nonlinear Sci. Numer. Simul. 14, 983-997 (2009)

15. Liao, S.J.: Beyond Perturbation: Introduction to the Homotopy Analysis Method. Chapman \& Hall/CRC Press, Boca Raton (2003)

16. Liao, S.J.: An optimal homotopy-analysis approach for strongly nonlinear differential equation. Commun. Nonlinear Sci. Numer. Simul. 15(8), 2003-2016 (2010)

17. Liao, S.J.: Homotopy Analysis Method in Nonlinear Differential Equations. Springer, Heidelberg (2012)

18. Pratibha, R., Jain, D., Prakash Saxena, V.: Approximate analytical solution with stability analysis of HIV/AIDS model. Cogent Math. 3, 1-14 (2016)

19. Ghoreishi, M., Md Ismail, A.I.B., Alomari, A.K.: Application of the homotopy analysis method for solving a model for HIV infection of CD4+ T-cells. Math. Comput. Model. 54, 3007-3015 (2011)

20. Alomari, A.K., Noorani, M.S.M., Nazar, R., Li, C.P.: Homotopy analysis method for solving fractional Lorenz system. Commun. Nonlinear Sci. Numer. Simul. 15(7), 1864-1872 (2010)

21. Bataineh, A.S., Noorani, M.S.M., Hashim, I.: Modified homotopy analysis method for solving systems of second-order BVPs. Commun. Nonlinear Sci. Numer. Simul. 14(2), 430-442 (2009)

22. Awawdeh, F., Adawi, A., Mustafa, Z:: Solutions of the SIR models of epidemics using HAM. Chaos Solitons Fractals $\mathbf{4 2}$ 3047-3052 (2009)

23. Kurulay, M.: Solving the fractional nonlinear Klein-Gordon equation by means of the homotopy analysis method. Adv. Differ. Equ. 2012, 187 (2012)

24. Perelson, A.S., Ribeiro, R.M.: Modeling the within-host dynamics of HIV infection. BMC Biol. 11, 96 (2013)

25. Neumann, A.U., Lam, N.P., Dahari, H., Gretch, D.R., Wiley, T.E., Layden, T.J., Perelson, A.S.: Hepatitis C viral dynamics in vivo and the antiviral efficacy of interferon-al pha therapy. Science 282(5386), 103-107 (1998)

26. Taylor, S.D., Leib, S.R., Wu, W., Nelson, R., Carpenter, S., Mealey, R.H.: Protective effects of broadly neutralizing immunoglobulin against homologous and heterologous equine infectious anemia virus infection in horses with severe combined immunodeficiency. J. Virol. 85(13), 6814-6818 (2011)

27. VandenDriessche, P., Watmough, J.: Reproduction numbers and sub-threshold endemic equilibria for compartmental models of disease transmission. Math. Biosci. 180, 29-48 (2002)

28. LaSalle, J.P.: The Stability of Dynamical Systems. CBMS-NSF Regional Conf. Ser. in Appl. Math., vol. 25. SIAM, Philadelphia (1976)

29. Shuai, Z., vandenDriessche, P.: Global stability of infectious disease models using Lyapunov functions. SIAM J. Appl. Math. 73(4), 1513-1532 (2013)

30. Schwartz, E.J., Vaidya, N.K., Dorman, K., Carpenter, S., Mealey, R.H.: Dynamics of lentiviral infection in vivo in the absence of adaptive host immune responses. Virology 513, 108-113 (2018)

31. Duarte, J., Januario, C., Martins, N., Correia Ramos, C., Rodrigues, C., Sardanyes, J.: Optimal homotopy analysis of a chaotic HIV-1 model incorporating AIDS-related cancer cells. Numer. Algorithms 77(1), 261-288 (2018)

32. Yabushita, K., Yamashita, M., Tsuboi, K.: An analytical solution of projectile motion with the quadratic resistance law using the homotopy analysis method. J. Phys. A, Math. Theor. 40, 8403-8416 (2007) 